\title{
NOD2 Regulates Hematopoietic Cell Function During Graft-versus-host Disease
}

\section{Citation}

Penack, Olaf, Odette M. Smith, Amy Cunningham-Bussel, Xin Liu, Uttam Rao, Nury Yim, Il-Kang $\mathrm{Na}$, et al. 2009. NOD2 regulates hematopoietic cell function during graft-versus-host disease. The Journal of Experimental Medicine 206(10): 2101-2110.

\section{Published Version}

doi:10.1084/jem.20090623

\section{Permanent link}

http://nrs.harvard.edu/urn-3:HUL.InstRepos:8160862

\section{Terms of Use}

This article was downloaded from Harvard University's DASH repository, and is made available under the terms and conditions applicable to Other Posted Material, as set forth at http:// nrs.harvard.edu/urn-3:HUL.InstRepos:dash.current.terms-of-use\#LAA

\section{Share Your Story}

The Harvard community has made this article openly available.

Please share how this access benefits you. Submit a story.

\section{Accessibility}




\section{NOD2 regulates hematopoietic cell function during graft-versus-host disease}

\author{
Olaf Penack, ${ }^{1,3}$ Odette M. Smith, ${ }^{1}$ Amy Cunningham-Bussel, ${ }^{4}$ Xin Liu, ${ }^{4}$ \\ Uttam Rao, ${ }^{1}$ Nury Yim, ${ }^{1}$ Il-Kang Na, ${ }^{1,3}$ Amanda M. Holland,,${ }^{1,4}$ \\ Arnab Ghosh, ${ }^{1}$ Sydney X. Lu, ${ }^{1}$ Robert R. Jenq, ${ }^{1,2}$ Chen Liu, ${ }^{5}$ \\ George F. Murphy, ${ }^{6}$ Katharina Brandl, ${ }^{1}$ and Marcel R.M. van den Brink ${ }^{1,2}$
}

'Department of Immunology and 'Department of Medicine, Memorial Sloan-Kettering Cancer Center, New York, NY 10065 ${ }^{3}$ Department of Hematology and Oncology, Charité, Campus Benjamin Franklin, Berlin 12200, Germany ${ }^{4}$ Department of Immunology and Microbial Pathogenesis, Weill Cornell Medical College, New York, NY 10065 ${ }^{5}$ Department of Pathology, Immunology, and Laboratory Medicine, University of Florida College of Medicine, Gainesville, FL 32610

${ }^{6}$ Program in Dermatopathology, Brigham and Women's Hospital, Harvard Medical School, Boston, MA 02115

Nucleotide-binding oligomerization domain 2 (NOD2) polymorphisms are independent risk factors for Crohn's disease and graft-versus-host disease (GVHD). In Crohn's disease, the proinflammatory state resulting from NOD2 mutations have been associated with a loss of antibacterial function of enterocytes such as paneth cells. NOD2 has not been studied in experimental allogeneic bone marrow transplantation (allo-BMT). Using chimeric recipients with NOD2 ${ }^{-1-}$ hematopoietic cells, we demonstrate that NOD2 deficiency in host hematopoietic cells exacerbates GVHD. We found that proliferation and activation of donor T cells was enhanced in NOD-deficient allo-BMT recipients, suggesting that NOD2 plays a role in the regulation of host antigen-presenting cells (APCs). Next, we used bone marrow chimeras in an experimental colitis model and observed again that NOD2 deficiency in the hematopoietic cells results in increased intestinal inflammation. We conclude that NOD2 regulates the development of GVHD through its inhibitory effect on host APC function.

\section{CORRESPONDENCE}

Marcel R.M. van den Brink:

m-van-den-brink@ski.mskcc.org

Abbreviations used: allo-BMT, allogenic BM transplantation; allo-HSCT, allogeneic hematopoietic stem cell transplantation; AML, acute myeloid leukemia; GVHD, graft-versus-host disease; MDP, muramyl dipeptide; MLN, mesenteric LN; MLR, mixed leukocyte reaction; NOD2, nucleotide-binding oligomerization domain 2; SNP, single nucleotide polymorphism; TCD-BM, T cell-depleted BM; TLR, toll-like receptor.
Nucleotide-binding oligomerization domain 2 (NOD2) belongs to the family of intracellular NOD-like receptors, which are present in various cell types (Bertin et al., 1999; Inohara et al., 2001). NOD2 has a C-terminal leucine-rich repeat region for sensing microbial products, a central nucleotide-binding domain (NACHT), and an N-terminal CARD (caspase activation and recruitment domain). NOD2 detects muramyl dipeptide (MDP), a molecule which is produced during the synthesis and degradation of peptidoglycan, a cell wall component of most bacteria (Inohara and Nunez, 2003). Stimulation of NOD2 induces recruitment of Rip2 (also called RICK) at the N-terminal CARD domain. Activation of Rip2 leads to subsequent steps that ultimately result in activation and nuclear translocation of NF- $\mathrm{\kappa B}$ and transcription of its target genes.

NOD2 has been studied in human Crohn's disease and in mouse models for inflammatory

K. Brandl's present address is Dept. of Genetics, the Scripps Research Institute, La Jolla, CA 92037. bowel disease. Single nucleotide polymorphisms (SNPs) near, or within, the NOD2 leucinerich repeat region (G908R, L1007insC, and $\mathrm{R} 702 \mathrm{~W}$ ) constitute genetic risk factors for the development of Crohn's disease (Hugot et al., 2001; Ogura et al., 2001). Mutations in the NACHT region of NOD2 are linked to other inflammatory diseases such as Blau syndrome (Miceli-Richard et al., 2001) and early onset sarcoidosis (Kanazawa et al., 2005). Several groups have investigated the role of NOD2 in experimental models for intestinal inflammation, which has led to the development of three hypotheses regarding the function of NOD2. Kobayashi et al. (2005) found greater susceptibility to enteral infection with Listeria monocytogenes and reduced expression of $\alpha$ defensins, antibacterial peptides produced by intestinal Paneth cells, in NOD2 $2^{-/-}$mice. Watanabe et al.

2009 Penack et al. This article is distributed under the terms of an Attribution-Noncommercial-Share Alike-No Mirror Sites license for the first six months after the publication date (see http://www.jem.org/misc/terms.shtml). After six months it is available under a Creative Commons License (Attribution-Noncommercial-Share Alike 3.0 Unported license, as described at http://creativecommons .org/licenses/by-nc-sa/3.0/). 
(2004, 2006) demonstrated increased sensitivity to experimental colitis in NOD2 $2^{-1-}$ mice, which was driven by $\mathrm{T}$ helper type 1 cells and increased IL-12 release. Maeda et al. (2005) created a knockout/knockin transgenic NOD2 mouse using the mouse equivalent to a human mutation (L1007insC) and found abundant IL-1 $\beta$ production in response to MDP. Collectively, these experimental data do not allow for one unified conclusion regarding the mechanism of NOD2mediated regulation of inflammatory diseases.

Recently, SNPs in the NOD2 gene locus have been linked to graft-versus-host disease (GVHD), which is the major complication after allogeneic hematopoietic stem cell transplantation (allo-HSCT). GVHD is a systemic inflammatory disease caused by alloreactive donor $\mathrm{T}$ cells and characterized by tissue damage in gut, liver, and skin. Holler et al. (2004, 2006) found an association between SNPs (alleles 8, 12 , and 13) of the NOD2 gene in the donor or host and a higher incidence of GVHD, as well as increased transplantrelated mortality. Interestingly, these findings were only partially confirmed by Elmaagacli et al. (2006), who also found an increased incidence of GVHD when NOD2 SNPs were present in both the donor and recipient but reduced incidence of GVHD when the NOD2 SNPs were present in the donor only. In addition, Mayor et al. (2007) connected NOD2 SNPs with an increased leukemia relapse rate after alloHSCT, which could not be confirmed by other investigators (Holler et al., 2008). Collectively, it appears that NOD2 SNPs of allo-HSCT recipients influence posttransplant immunity; however, the underlying mechanism is unclear. In the present study, we aim to clarify the underlying role of NOD2 in mouse models for allogenic BM transplantation (allo-BMT), colitis, and tumors.

\section{RESULTS AND DISCUSSION \\ NOD2 deficiency in donor BM cells and/or donor T cells does not affect the development of GVHD}

To address contradictory clinical studies showing that NOD2 SNPs of the allo-HSCT donor can either exacerbate GVHD (Holler et al., 2004, 2006) or ameliorate GVHD (Elmaagacli et al., 2006), we investigated the effect of NOD2 deficiency of allo-BMT donors in our GVHD models. We first assessed whether NOD2 deficiency affects allogeneic donor $\mathrm{T}$ cells. We transferred CFSE-labeled B6 WT or B6 NOD2 $2^{-1-}$ $T$ cells to lethally irradiated $\mathrm{BALB} / \mathrm{c}$ recipients and found similar T cell proliferation (Fig. $1 \mathrm{~A}$ ), T cell activation (Fig. $1 \mathrm{~B}$ ), and expression of the gut-homing molecule LPAM (Fig. 1 C). Next, we performed mixed leukocyte reactions (MLRs) using $\mathrm{CD}^{+}{ }^{-}$-selected $\mathrm{B} 6 \mathrm{WT}$ or B6 $\mathrm{NOD}^{-1-}$ splenocytes as effectors and irradiated $\mathrm{BALB} / \mathrm{c}$ splenocytes as stimulators. We found no significant differences in the proliferation rate between WT T cells and NOD $2^{-/-} \mathrm{T}$ cells (Fig. $\left.1 \mathrm{D}\right)$. In addition, we found no significant differences in the proliferation rate between WT $\mathrm{T}$ cells and NOD2 $2^{-1-} \mathrm{T}$ cells when we added different toll-like receptor (TLR) and NOD2 ligands (MDP, LPS, Pam3Cys, Flagellin, and PGN) alone or in combination with the MLRs (unpublished data). To deter- mine the effect of NOD2 deficiency in allo-BMT donors on the development of GVHD, we first used an MHC-disparate allo-BMT model, B6 (H-2 $\left.2^{\mathrm{b}}\right) \rightarrow \mathrm{BALB} / \mathrm{c}\left(\mathrm{H}-2^{\mathrm{d}}\right)$. Lethally irradiated recipients received $\mathrm{T}$ cell-depleted $\mathrm{BM}$ (TCD-BM), and GVHD was induced by the addition of donor splenic $\mathrm{T}$ cells to the allograft. We used NOD $2^{-/-}$donors and WT donors as the source for TCD-BM or T cells. We did not detect significant differences in lethal GVHD between the four different groups receiving WT BM + WT T cells, WT $\mathrm{BM}+\mathrm{NOD}^{-/-} \mathrm{T}$ cells, NOD2 $2^{-/-} \mathrm{BM}+\mathrm{WT} \mathrm{T}$ cells, or NOD2 $2^{-1-}$ BM + NOD2 $2^{-1-}$ T cells (Fig. 1 E, left). To confirm that our observations were not strain dependent or model dependent, we performed similar experiments in an MHCmatched model with minor histocompatibility disparities, B6 $\left(\mathrm{H}-2^{\mathrm{b}}\right) \rightarrow \mathrm{LP}\left(\mathrm{H}-2^{\mathrm{b}}\right)$ (Fig. $1 \mathrm{E}$, right). We conclude that NOD2 deficiency of the allo-BMT donor (either donor $\mathrm{T}$ cells or BM) has no significant impact on the development of GVHD and does not regulate alloactivation of donor $\mathrm{T}$ cells. Our data suggest that NOD2 has no cell-intrinsic role in the regulation of $\mathrm{T}$ cell activity during GVHD despite the increasing evidence that innate immune receptors, such as TLRs, can modulate $\mathrm{T}$ cell function and activation during inflammation (Dabbagh and Lewis, 2003; Caron et al., 2005; MacLeod and Wetzler, 2007).

\section{NOD2-/- allo-BMT recipients have increased systemic and target organ GVHD}

To investigate the role of NOD2 in regulating GVHD in allo-BMT recipients, we first used the $\mathrm{MHC}$-disparate $\mathrm{B} 10 \mathrm{BR}\left(\mathrm{H}-2^{\mathrm{k}}\right) \rightarrow \mathrm{B} 6\left(\mathrm{H}-2^{\mathrm{b}}\right)$ model. We found significantly more lethal GVHD in B6 NOD2 $2^{-/-}$allo-BMT recipients as compared with B6 WT allo-BMT recipients (Fig. 2 A, left). We confirmed these findings in the MHC-matched LP $\left(\mathrm{H}-2^{\mathrm{b}}\right) \rightarrow \mathrm{B} 6\left(\mathrm{H}-2^{\mathrm{b}}\right)$ model (Fig. $2 \mathrm{~A}$, right). To analyze target organ GVHD, we performed histopathological analyses and found significantly more GVHD in the terminal ileum, colon, and liver, as well as a trend toward more skin GVHD in NOD2 $2^{-/-}$versus WT allo-BMT recipients (Fig. 2 B). The numbers of tissue-infiltrating alloreactive $\mathrm{CD}^{+} \mathrm{T}$ cells in GVHD target organs were increased in NOD2 $2^{-1-}$ allo-BMT recipients (Fig. 2, C and D). To investigate thymic GVHD, we determined the number of thymocytes and their subsets at day 21 after allo-BMT. We found a significantly reduced number of $\mathrm{CD}^{+} / \mathrm{CD}^{+}$double-positive thymocytes in NOD2 $2^{-/-}$allo-BMT recipients (Fig. $2 \mathrm{E}$ ), which is the characteristic feature of thymic GVHD (Hollander et al., 1994). We conclude that NOD2 deficiency in allo-BMT recipients exacerbates systemic and organ GVHD. Our results are in agreement with clinical data connecting NOD2 SNPs in allo-BMT recipients to increased incidence and severity of GVHD (Holler et al., 2004, 2006). A limitation of our study is that in humans the association between NOD2 and GVHD was observed in individuals with heterozygous SNPs, whereas our studies used homozygous NOD2-deficient allo-BMT recipients to investigate the effect of NOD2 deficiency during GVHD. This is similar to studies in mice and men regarding 
the role of NOD2 in Crohn's disease (Hugot et al., 2001; Watanabe et al., 2004, 2006, 2008; Kobayashi et al., 2005). NOD2 polymorphisms resulting in impaired NOD2 function were associated with increased susceptibility to Crohn's disease. Studies in NOD2-deficient mice demonstrated an increased susceptibility to experimental colitis, whereas mice with a NOD2 transgene resulting in increased NOD2 function were resistant to experimental colitis.

The activation and proliferation of alloreactive donor $\mathrm{T}$ cells is increased in NOD2 ${ }^{-1-}$ allo-BMT recipients

To study the mechanisms underlying increased GVHD in $\mathrm{NOD}^{-/-}$allo-BMT recipients, we first transferred CFSE- labeled allogeneic B10BR $\mathrm{T}$ cells into B6 WT or B6 $\mathrm{NOD}^{-/-}$allo-BMT recipients. We found that the in vivo proliferation of alloreactive donor $\mathrm{T}$ cells was significantly increased in NOD2 $2^{-/-}$allo-BMT recipients (Fig. 2 F). Next, we performed allo-BMTs in the $\mathrm{B} 10 \mathrm{BR} \rightarrow \mathrm{B} 6$ model and harvested organs at day 14 after allo-BMT. We found that the number of donor $\mathrm{T}$ cells was significantly increased in spleen and mesenteric LNs (MLNs) in NOD2 $2^{-/-}$allo-BMT recipients (Fig. $2 \mathrm{G}$, left). However, the percentages of $\mathrm{CD}^{+}$ $\mathrm{T}$ cells, CD8 ${ }^{+} \mathrm{T}$ cells, and CD44 ${ }^{+} / \mathrm{CD} 62 \mathrm{~L}^{-}$effector $\mathrm{T}$ cells were not significantly different (Fig. S1). We next determined the alloactivation status of donor T cells by their CD25 expression, which is a reliable marker for alloactivation in our
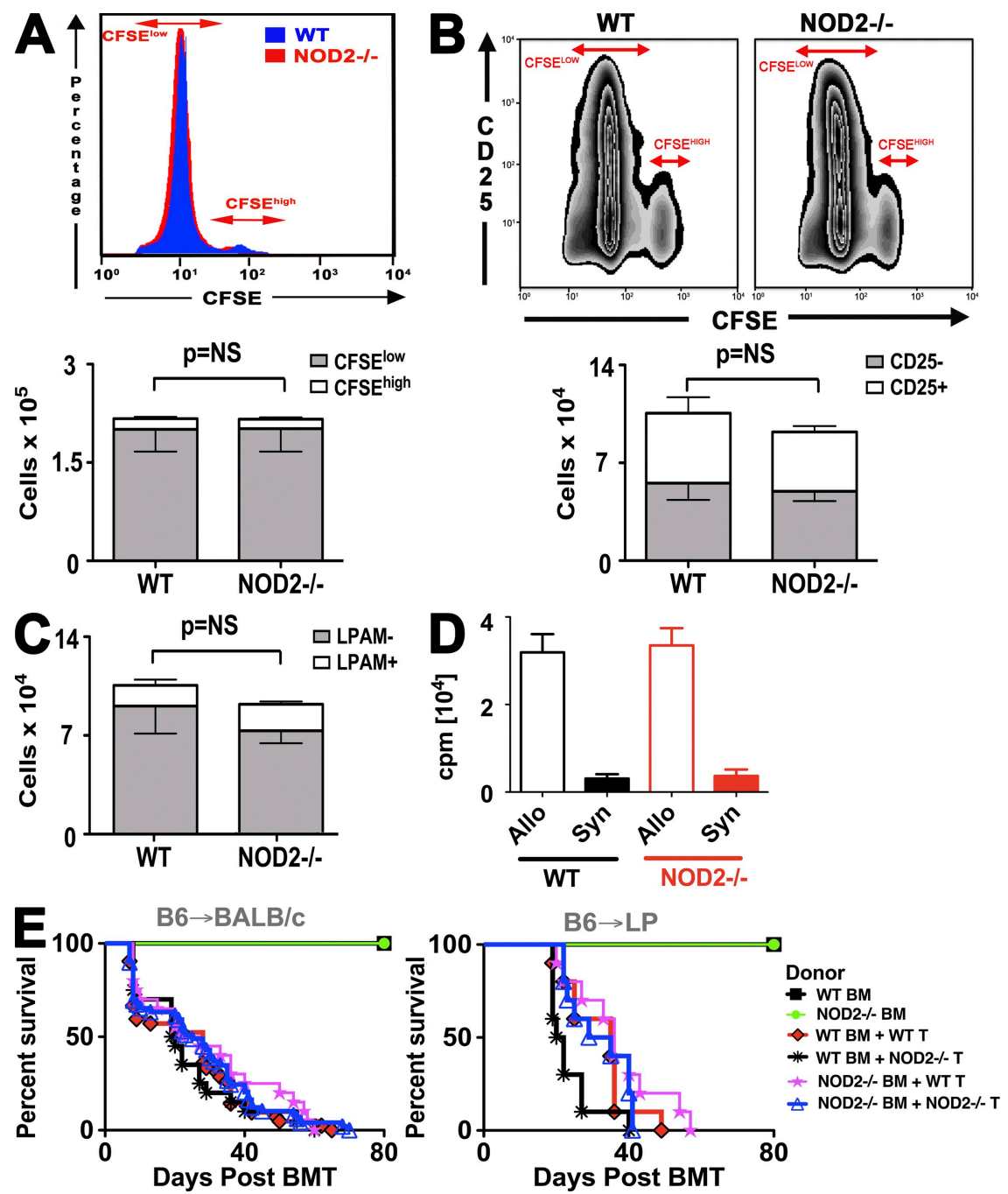

Figure 1. NOD2 deficiency in donor BM cells and/or donor T cells does not affect the development of GVHD. (A-C) Lethally irradiated (8.5 Gy) BALB/c allo-BMT recipients received $5 \times 10^{6}$ CFSE-labeled B6 WT or B6 NOD2 ${ }^{-1-C D 5^{+}}$T cells. Recipient spleens were harvested $96 \mathrm{~h}$ after transfer of T cells; $n=8 /$ group; combined data from two independent experiments are shown. (A) Identical CFSE histogram overlays of WT and NOD2-1- donor T cells (left), as well as similar total number of CFSEhigh and CFSElow donor T cells. (B) Similar up-regulation of the alloactivation marker CD25 on WT B6 donor T cells versus NOD2-I- donor T cells. (C) Similar expression of the gut-homing marker LPAM on WT B6 donor T cells versus NOD2 ${ }^{-1-}$ donor T cells. (D) MLR with WT versus NOD2 ${ }^{-1-}$ CD5 ${ }^{+}$T cells and irradiated BALB/c stimulators. Combined data of three independent experiments are shown; $n=12 / g r o u p$. (E) Lethally irradiated BALB/C (8.5 Gy) or LP (11Gy) allo-BMT recipients were transplanted with $5 \times 10^{6}$ B6 WT TCD-BM versus B6 NOD2 ${ }^{-1-}$ TCD-BM and $10^{6}$ B6 WT T cells versus B6 NOD2 ${ }^{-1-}$ T cells. Combined data from four independent experiments are shown; $n=15-45 / g r o u p$. Error bars indicate SEM. 

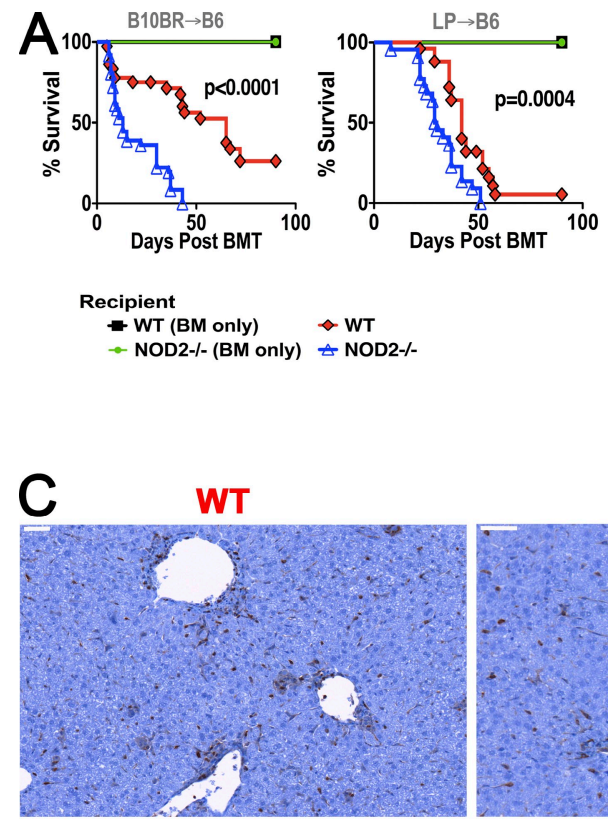

$\mathrm{E}$

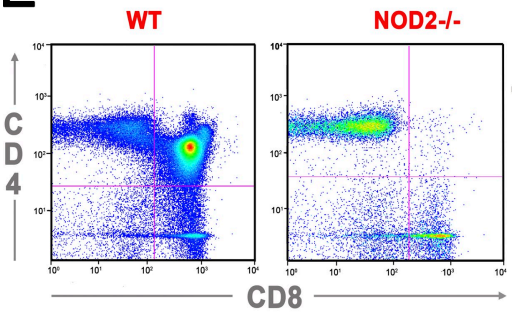

G Spleen

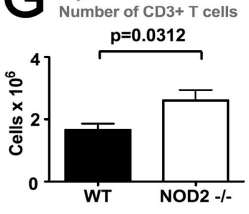

MLN
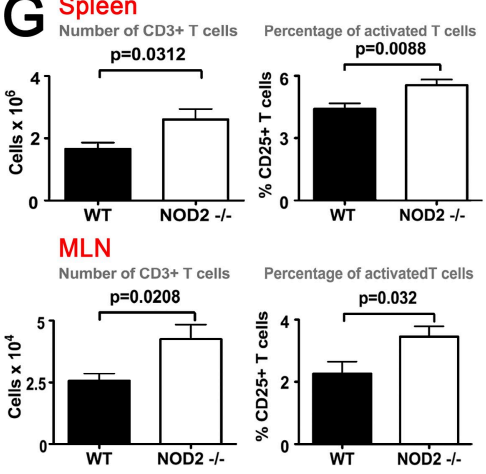
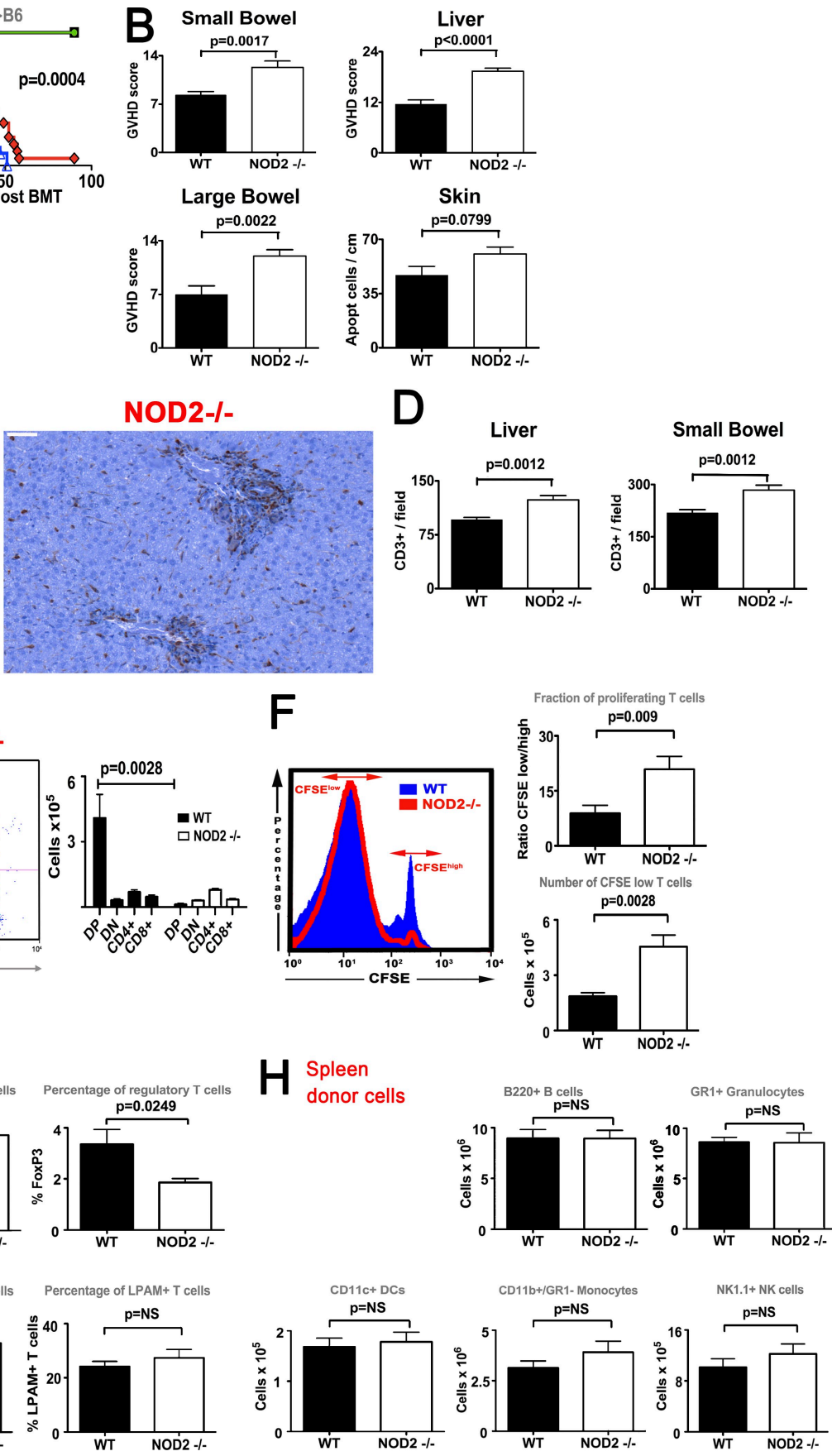

Figure 2. NOD2-I- allo-BMT recipients have increased systemic and target organ GVHD because of enhanced activation and proliferation of alloreactive donor T cells. (A) Lethally irradiated (11 Gy) B6 WT versus B6 NOD2-1- allo-BMT recipients received $5 \times 10^{6}$ B10BR TCD-BM or $5 \times 10^{6}$ LP TCD-BM with or without $10^{6}$ B10BR T cells or $2 \times 10^{6} \mathrm{LPT}$ cells. Combined data from four independent experiments are shown; $n=45 / \mathrm{group}$. (B-E) Lethally irradiated (11 Gy) B6 WT versus B6 NOD2 ${ }^{-1-}$ allo-BMT recipients received $5 \times 10^{6}$ B10BR TCD-BM $+10^{6}$ B10BR T cells. Organs were harvested at day 21 after allo-BMT. Combined data from two independent experiments are shown; $n=10 / g r o u p$. (B) Histopathologic GVHD scores in target organs showing increased GVHD in NOD2 ${ }^{-1-}$ allo-BMT recipients. (C) CD3+ histochemistry images showing increased infiltration of T cells (brown) in liver tissue of NOD2 ${ }^{-1-}$ allo-BMT recipients. Bars, $50 \mu \mathrm{m}$. (D) Quantification of T cell infiltration in GVHD target organs by $\mathrm{CD}^{+}{ }^{+}$histochemistry. (E) Increased thymic GVHD in NOD2 ${ }^{-1-}$ allo-BMT recipients demonstrated by reduced numbers of $\mathrm{CD} 4^{+} / \mathrm{CD} 8^{+}$double-positive thymocytes. Shown are flow cytometric plots and the absolute numbers of DP $\left(\mathrm{CD} 4^{+} / \mathrm{CD} 8^{+}\right)$, DN $\left(\mathrm{CD} 4^{-} / \mathrm{CD} 8^{-}\right), \mathrm{CD} 4^{+}$, and $\mathrm{CD} 8^{+}$thymocytes. (F) Lethally irradiated (11 Gy) B6 WT versus B6 N0D2 ${ }^{-1-}$ allo-BMT recipients received $5 \times 10^{6} \mathrm{CFSE}-$ labeled B10BR CD5+ T cells. Recipient spleens were harvested $96 \mathrm{~h}$ after transfer of T cells; $n=8 / \mathrm{group} ;$ combined data from two independent experiments are shown. CFSE histogram overlays of donor T cells in WT allo-BMT recipients versus NOD2-1- allo-BMT recipients (left); ratio of proliferating donor T cells (right, top) and absolute numbers of donor T cells (right, bottom) showing increased donor T cell proliferation in NOD2-1- allo-BMT recipients. ( $\mathrm{G}$ and $\mathrm{H}$ ) Lethally irradiated (11 Gy) B6 WT versus B6 NOD2-1- allo-BMT recipients were transplanted with 
GVHD models (Alpdogan et al., 2003), and found that donor $\mathrm{T}$ cells were significantly more activated in NOD2 $2^{-/-}$alloBMT recipients as compared with $\mathrm{WT}$ allo-BMT recipients (Fig. $2 \mathrm{G}$, middle). When we analyzed FoxP3 ${ }^{+} / \mathrm{CD}^{+}$regulatory $\mathrm{T}$ cells in the spleen, we found a significantly reduced percentage of FoxP3 ${ }^{+} / \mathrm{CD}^{+}{ }^{+}$cells (of total $\mathrm{CD}^{+}{ }^{+}$splenocytes) in $\mathrm{NOD}^{-/-}$allo-BMT recipients (Fig. $2 \mathrm{G}$, top right). The percentages of $\mathrm{IL}-17^{+} \mathrm{CD} 4^{+}, \mathrm{IFN}-\gamma^{+} \mathrm{CD} 4^{+}$, and $\mathrm{Tbet}^{+} \mathrm{CD} 4^{+}$ $\mathrm{T}$ cells were not different (Fig. S1). There was no difference between the expression of the gut-homing molecule LPAM ( $\alpha 4 \beta 7$ integrin) on donor T cells in MLNs in NOD2 ${ }^{-/-}$and WT allo-BMT recipients (Fig. $2 \mathrm{G}$, bottom right). We detected no differences in donor cell types, such as B cells, NK cells, granulocytes, monocytes, and DCs in NOD2 $2^{-/-}$allo$\mathrm{BMT}$ recipients (Fig. $2 \mathrm{H}$ ). We conclude that increased GVHD in NOD2 $2^{-/-}$allo-BMT recipients is the result of enhanced alloactivation and proliferation of donor $\mathrm{T}$ cells.

\section{NOD2 deficiency in the hematopoietic system of the allo-BMT recipient exacerbates GVHD}

Given that NOD2 is expressed in both hematopoietic and nonhematopoietic cells types, including antigen-presenting cells and intestinal epithelial cells, respectively, it is not clear what relative contributions of these cells types is to the development of GVHD. To address this, we created BM chimeras in which the NOD2 deficiency was only manifest in one of these systems. BM chimeras were created by performing syngeneic BMTs after lethal irradiation, using B6 WT and $\mathrm{B} 6 \mathrm{NOD} 2^{-/-}$as donors or as recipients $\left(\mathrm{WT} \rightarrow \mathrm{NOD} 2^{-/-}\right.$or $\left.\mathrm{NOD}^{-/-} \rightarrow \mathrm{WT}\right)$. Using B6 Ly5.1 ${ }^{+}$mice as donors, we found the donor chimerism of hematopoietic cell subsets to be $>95 \%$ in GVHD target organs, as well as in secondary lymphoid organs at day 90 after syngeneic BMT (Fig. S2). We then used the BM chimera, WT mice, and NOD2 $2^{-1-}$ mice as allo-BMT recipients in the $\mathrm{B} 10 \mathrm{BR} \rightarrow \mathrm{B} 6 \mathrm{GVHD}$ model. We found that $\mathrm{NOD}^{-/-}$and $\mathrm{NOD} 2^{-/-} \rightarrow \mathrm{WT}$ chimeric alloBMT recipients had a greater GVHD-induced mortality rate as compared with WT and $\mathrm{WT} \rightarrow \mathrm{NOD} 2^{-/-}$chimeric allo$\mathrm{BMT}$ recipients (Fig. $3 \mathrm{~A}$, left). We confirmed our findings in the minor mismatched $\mathrm{LP} \rightarrow \mathrm{B} 6$ model (Fig. $3 \mathrm{~A}$, right). To analyze target organ GVHD, we performed histopathological analyses and found significantly more GVHD in the terminal ileum, colon, liver, and skin in $\mathrm{NOD}^{-/-} \rightarrow \mathrm{WT}$ chimeric allo-BMT recipients versus $\mathrm{WT} \rightarrow \mathrm{NOD}^{-/-}$chimeric allo-BMT recipients (Fig. $3 \mathrm{~B}$ ). The numbers of tissueinfiltrating $\mathrm{CD}^{+} \mathrm{T}$ cells in GVHD target organs were increased in $\mathrm{NOD}^{-/-} \rightarrow \mathrm{WT}$ chimeric allo-BMT recipients (Fig. $3 \mathrm{C}$ ). We found that the number of donor $\mathrm{T}$ cells was significantly increased in the spleen and MLNs in NOD2 $2^{-/-} \rightarrow$ WT chimeric allo-BMT recipients (Fig. 3 D, left). We next determined the alloactivation status of donor $\mathrm{T}$ cells by their CD25 expression and found that donor T cells were significantly more activated in NOD2 $2^{-/-} \rightarrow \mathrm{WT}$ chimeric allo-BMT recipients as compared with $\mathrm{WT} \rightarrow \mathrm{NOD} 2^{-/-}$chimeric alloBMT recipients (Fig. 3 D, middle). Additionally, we found a significantly reduced percentage of $\mathrm{FoxP}^{+} / \mathrm{CD}^{+}{ }^{+}$regulatory $\mathrm{T}$ cells in the spleen (percentage of $\mathrm{FoxP}^{+}$in total $\mathrm{CD}^{+}$ splenocytes) in NOD $2^{-/-} \rightarrow$ WT chimeric allo-BMT recipients (Fig. 3 D, top right). The expression of LPAM on donor $\mathrm{T}$ cells in MLNs was similar in both chimeric allo-BMT recipients (Fig. 3 D, bottom right). We conclude that NOD2 deficiency in the hematopoietic system exacerbates GVHD, whereas NOD2 deficiency in the nonhematopoietic system has no significant impact on the development of GVHD in NOD $2^{-/-}$allo-BMT recipients.

\section{NOD2 ${ }^{-1-}$ DCs have a higher activation status and increased ability to induce T cell proliferation during GVHD}

Based upon our finding that donor $\mathrm{T}$ cell proliferation is increased in allo-BMT recipients with NOD2 deficiency in the hematopoietic system, we hypothesized that NOD2 ${ }^{-1-}$ DCs might have a higher activation status and increased ability to induce $\mathrm{T}$ cell proliferation during GVHD. We therefore performed allo-BMTs in the B10BR $\rightarrow$ B6 model and harvested spleens at day 7 after allo-BMT. We found that the absolute numbers of host DC subsets in the spleen were not significantly different before and after allo-BMT in WT versus NOD2 $2^{-/-}$ allo-BMT recipients (Fig. 4 A). We then quantified the expression of the activation markers and costimulatory molecules CD40, CD80, and CD86 on host $\left(\mathrm{CD} 11 \mathrm{c}^{+} \mathrm{H} 2 \mathrm{~kb}^{+}\right)$DCs before allo-BMT and at day 7 after allo-BMT. We found a significantly increased up-regulation of activation markers on host NOD2 $2^{-1-}$ DCs in contrast to WT DCs during GVHD (Fig. 4, B and C). The increase in the expression of $\mathrm{MHC}$ class II molecules, which was already high at baseline, did not reach statistical significance (unpublished data). To study DC function, we selected splenic DCs from WT and NOD2 $2^{-/-}$ allo-BMT recipients with GVHD (day 7) and used them as stimulators in MLRs. We found that B6 NOD2 $2^{-/-}$DCs had a significantly increased ability to induce proliferation of allogeneic B10BR T cells as compared with B6 WT DCs (Fig. 4 D). These data supports the hypothesis that NOD2 can negatively regulate the activity and function of host DCs, resulting in increased alloactivation and proliferation of donor $\mathrm{T}$ cells in NOD2 $2^{-/-}$allo-BMT recipients. Our results provide a possible cellular mechanism for recent clinical studies suggesting a regulatory role of NOD2 on the incidence of GVHD and transplant-related mortality in allo-HSCT recipients with SNPs in the gene locus encoding NOD2 (Holler et al., 2004, 2006; Elmaagacli et al., 2006).

$5 \times 10^{6}$ B10BR TCD-BM $+10^{6}$ B10BR T cells. Organs were harvested at day 14 after allo-BMT. Combined data from two independent experiments are shown; $n=10 /$ group. (G) Absolute numbers and activation status (CD25+) of donor CD3 ${ }^{+} \mathrm{T}$ cells in spleen and MLNs. Percentage of FoxP3 ${ }^{+}$cells in CD4+ splenocytes (top right) and expression of the gut homing marker LPAM in MLNs (bottom right) are shown. (H) Absolute numbers of donor B cells, NK cells, monocytes, DCs, and granulocytes in spleen. Error bars indicate SEM. 
In previous studies using NOD $2^{-/-}$mice in inflammatory bowel disease models, it was suggested that increased TLR signaling led to a massively increased production of cytokines such as IL-12. (Watanabe et al., 2004, 2006, 2008; Strober et al., 2006; Yang et al., 2007) To determine whether increased cytokine/chemokine production is the underlying mechanism for the enhanced ability of NOD2 $2^{-/-}$DCs to induce $\mathrm{T}$ cell proliferation, we performed allo-BMTs in the $\mathrm{B} 10 \mathrm{BR} \rightarrow \mathrm{B} 6$ model and measured cytokines/chemokines in serum with a multiplex system at day 7 after allo-BMT. We did not detect significant differences in serum levels of IFN- $\gamma$, IL-1 $\alpha$, IL-1 $\beta$, IL-2, IL-6, IL-7, IL-9, IL-10, IL-12 p40,
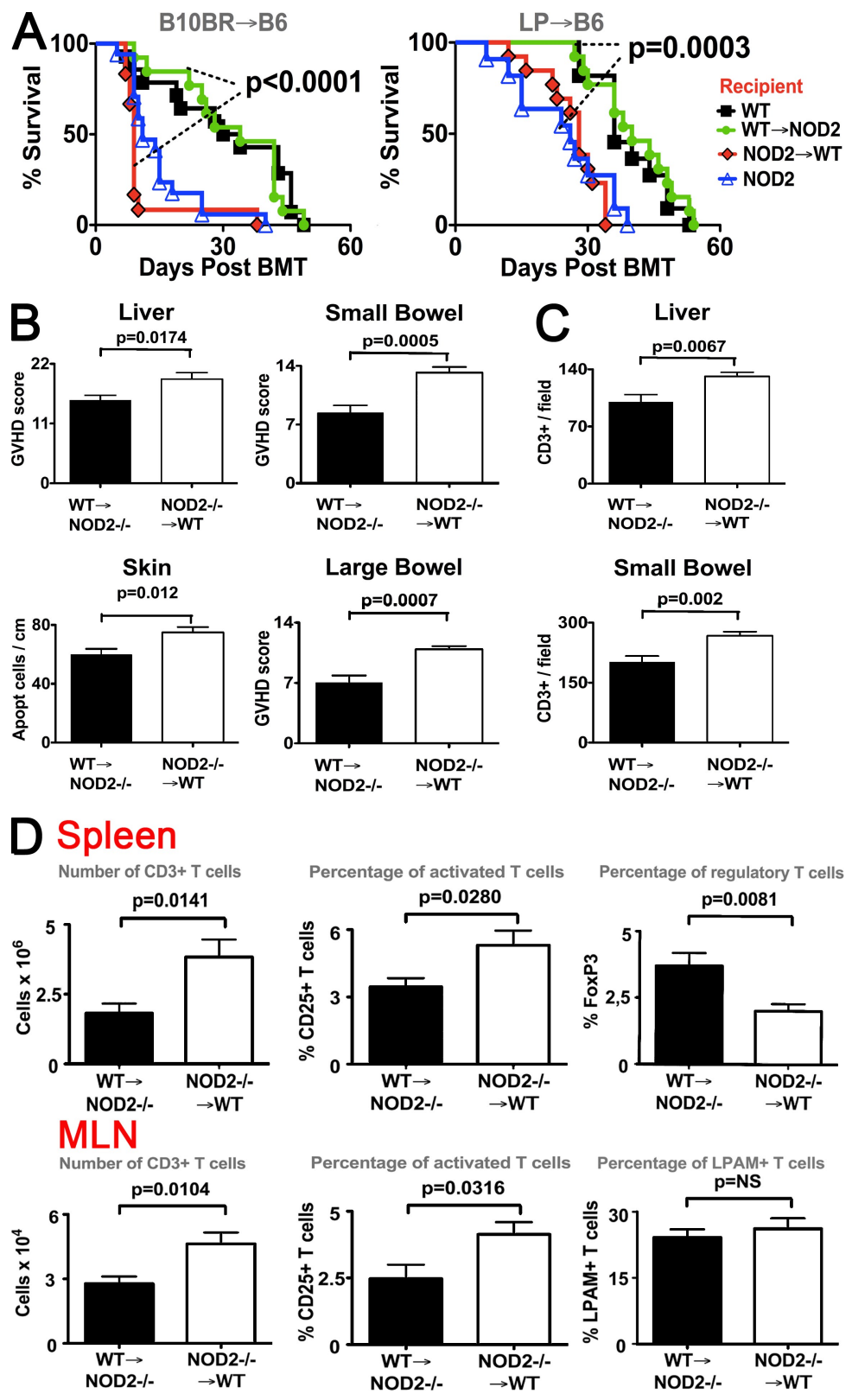

Figure 3. NOD2 deficiency of the hematopoietic system of the allo-BMT recipient aggravates GVHD. (A-D) Chimeric mice with NOD2 deficiency either in the hematopoietic system or in the nonhematopoietic system were created by syngeneic BMT (B6 WT $\rightarrow$ B6 NOD2 $2^{-1-}$ or B6 NOD2 $2^{-1-} \rightarrow$ B6 WT). After 90 d, lethally irradiated (11 Gy) B6 WT versus B6 NOD2-1- versus chimeric allo-BMT recipients were transplanted with $5 \times 10^{6}$ B10BR TCD-BM or $5 \times 10^{6} \mathrm{LP} \mathrm{TCD}-\mathrm{BM}+2 \times 10^{6} \mathrm{~B} 10 \mathrm{BR}$ T cells or $3 \times 10^{6} \mathrm{LP}$ T cells. (A) Survival curves. Combined data from two independent experiments are shown; $n=16 /$ group. (B-E) Lethally irradiated (11 Gy) B6 WT $\rightarrow$ B6 NOD2 ${ }^{-1-}$ or B6 NOD2-1- $\rightarrow$ B6 WT chimeric allo-BMT recipients were transplanted with $5 \times 10^{6}$ B10BR TCD-BM $+10^{6}$ B10BR T cells. Organs were harvested at day 14 after allo-BMT. Combined data from two independent experiments are shown; $n=8 /$ group. (B) Histopathologic GVHD scores in GVHD target organs. (C) Quantification of T cell infiltration in GVHD target organs by CD3+ histochemistry. (D) Absolute number and activation status (CD25+) of donor $\mathrm{CD} 3^{+}$T cells in spleen and MLNs, percentage of FoxP3 ${ }^{+}$regulatory T cells (top right), and expression of the gut-homing marker LPAM in MLNs (bottom right). Error bars indicate SEM. 
IL-12 p70, IL-15, IL-17, IP-10, LIX, MCP-1, MIG, MIP-1 $\alpha$, MIP-1 $\beta$, Rantes, and TNF in NOD2 ${ }^{-1-}$ allo-BMT recipients versus WT allo-BMT recipients (Fig. S3). Although serum levels were not different we cannot exclude differences
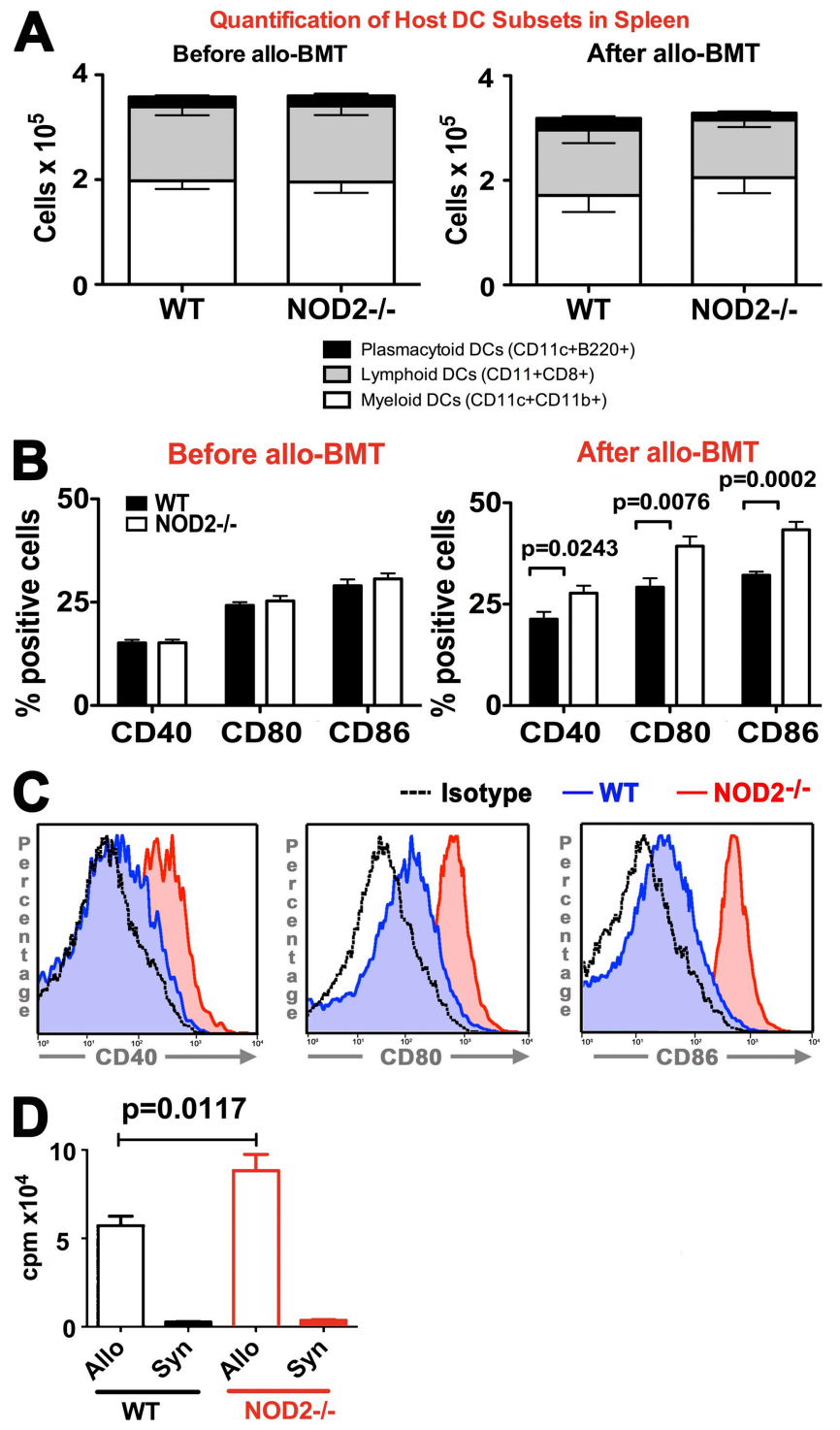

Figure 4. NOD2-l- DCs have a higher activation status and increased ability to induce T cell proliferation during GVHD. Lethally irradiated (11 Gy) B6 WT versus B6 NOD2-l- allo-BMT recipients were transplanted with $5 \times 10^{6}$ B10BR TCD-BM $+2 \times 10^{6}$ B10BR T cells. Organs were harvested at day 7 after allo-BMT. Donor and host-derived DCs were distinguished by $\mathrm{MHC}$ class I disparity in flow cytometry $\left(\mathrm{H} 2^{k}\right.$ vs. $\left.\mathrm{H} 2^{b}\right)$. Combined data from two independent experiments are shown; $n=8 /$ group. (A) Quantification of host DC subsets by flow cytometry. (B) Quantification of activation marker expression of host DCs by flow cytometry before allo-BMT and at day 7 after allo-BMT. (C) Histogram overlays of activation marker expression of host DCs during GVHD. (D) MLR using $10^{5}$ splenic DCs (CD11+ selection) from B6 WT allo-BMT recipients versus B6 NOD2-l- allo-BMT recipients as stimulators and $10^{5}$ B10BR T cells (CD5+ selection). Shown is the thymidine $\mathrm{H}^{3}$ uptake (cpm) between 48 and $72 \mathrm{~h}$ of MLR. Error bars indicate SEM. in cytokine production in specified cell populations. However, our results are in agreement with recent data, which showed that APCs from humans with NOD2 SNPs do not have increased cytokine production (van Heel et al., 2005). Consequently, there might be alternative molecular mechanisms that can enhance APC function and activity in $\mathrm{NOD} 2^{-/-}$ allo-BMT recipients during inflammatory diseases such as colitis and GVHD.

\section{NOD2 deficiency of the hematopoietic system exacerbates} experimental colitis

Next, we analyzed whether results from our GVHD experiments also apply to other inflammatory diseases. Because the pathophysiology of GVHD and inflammatory bowel disease has many similarities, we performed experiments using the TNBS (2,4,6-trinitrobenzenesulfonic acid) colitis model. TNBS colitis is a transmural inflammation often used as a model for inflammatory bowel disease (Scheiffele and Fuss, 2002). Administration of TNBS leads to modification of intestinal proteins that function as haptens. We rectally challenged B6 WT or B6 NOD2 $2^{-1-}$ mice with $5 \mathrm{mg}$ TNBS in $50 \%$ ethanol. We found a significantly higher weight loss in $\mathrm{NOD}^{-/-}$mice than in WT mice during colitis (Fig. 5 A). We next compared the NOD2 $2^{-1-} \rightarrow \mathrm{WT}$ chimeric to the $\mathrm{WT} \rightarrow \mathrm{NOD} 2^{-1-}$ chimeric mice ( $90 \mathrm{~d}$ after syngeneic BMT) in the TNBS colitis model. We found significantly more weight loss in $\mathrm{NOD} 2^{-\prime-} \rightarrow \mathrm{WT}$ chimeric mice versus the $\mathrm{WT} \rightarrow \mathrm{NOD} 2^{-/-}$ chimeric mice (Fig. 5 B). We harvested colons at day 3 after induction of colitis, performed histopathological analysis, and found significantly higher colitis scores in NOD2 $2^{-/} \rightarrow \mathrm{WT}$ chimeric mice as compared with the $\mathrm{WT} \rightarrow \mathrm{NOD} 2^{-1-}$ chimeric mice (Fig. 5, C and D). Our data demonstrate that NOD2 deficiency in the hematopoietic system, but not in the nonhematopoietic system, exacerbates both experimental GVHD and experimental TNBS colitis. These results suggest that loss of intestinal epithelial cell function causing impaired antibacterial resistance, which has been proposed as a mechanism for increased inflammation in NOD2 $2^{-/-}$mice (Kobayashi et al., 2005), is not responsible for the increased GVHD and colitis. Our results are in agreement with previous findings (Watanabe et al., 2004, 2006, 2008; Strober et al., 2006; Yang et al., 2007) that found increased susceptibility to different types of experimental colitis in NOD2 $2^{-1-}$ mice, which was the result of the enhanced ability of NOD $2^{-/-}$APCs to trigger inflammatory $\mathrm{T}$ cell responses.

\section{NOD2 ${ }^{-I-}$ mice have intact resistance against experimental tumors}

In response to mixed clinical results on a possible role of NOD2 in antitumor immunity (Mayor et al., 2007; Holler et al., 2008), we performed tumor challenges with hematologic and solid tumors. We studied the role of NOD2 in antitumor responses in two tumor models: acute myeloid leukemia (AML; C1498) and melanoma (B16). When we challenged B6 WT or B6 NOD2 $2^{-/-}$mice intravenously with C1498 or B16, we found no significant differences in survival (unpublished 
data). Bioluminescence imaging demonstrated that early tumor growth was slightly reduced in $\mathrm{B} 6 \mathrm{NOD} 2^{-/-}$mice in both models (Fig. S4). We conclude that NOD2 $2^{-1-}$ has no major role in antitumor immunity against C1498 AML and B16 melanoma.

\section{MATERIALS AND METHODS}

Mice and allo-BMT. Animal protocols were approved by the Memorial SloanKettering Cancer Center Institutional Animal Care and Use Committee. NOD2 $2^{-1-}$ mice (Kobayashi et al., 2005) were backcrossed to a B6 background $(n=11)$. BMT experiments and bioluminescent imaging was performed as described previously (Terwey et al., 2005; Zakrzewski et al., 2006, 2008).
Flow cytometry and MLR. Most antibodies were obtained from BD. DCs were purified by positive selection with CD11c (N418) MicroBeads (Miltenyi Biotec) from digested spleens. Purity was between 90 and $95 \%$. Splenic T cells were enriched by $\mathrm{CD}^{+}$selection and cocultured. After $48 \mathrm{~h}$, thymidine $\left(1 \mu \mathrm{Ci}[0.037 \mathrm{MBq}]\left[{ }^{3} \mathrm{H}\right]\right.$ thymidine/well) was added and, after $72 \mathrm{~h}$, thymidine uptake was measured (TopCount; Packard).

Proliferation of CFSE-labeled $\mathrm{T}$ cells in vivo. T cells were selected using CD5 MicroBeads (Miltenyi Biotec), stained with $5 \mu \mathrm{M}$ CFSE (Invitrogen), and transplanted into lethally irradiated allogeneic recipients.

Measurement of cytokines and chemokines. Serum levels were determined using the Milliplex Mouse Cytokine/Chemokine Immunoassay (Millipore).
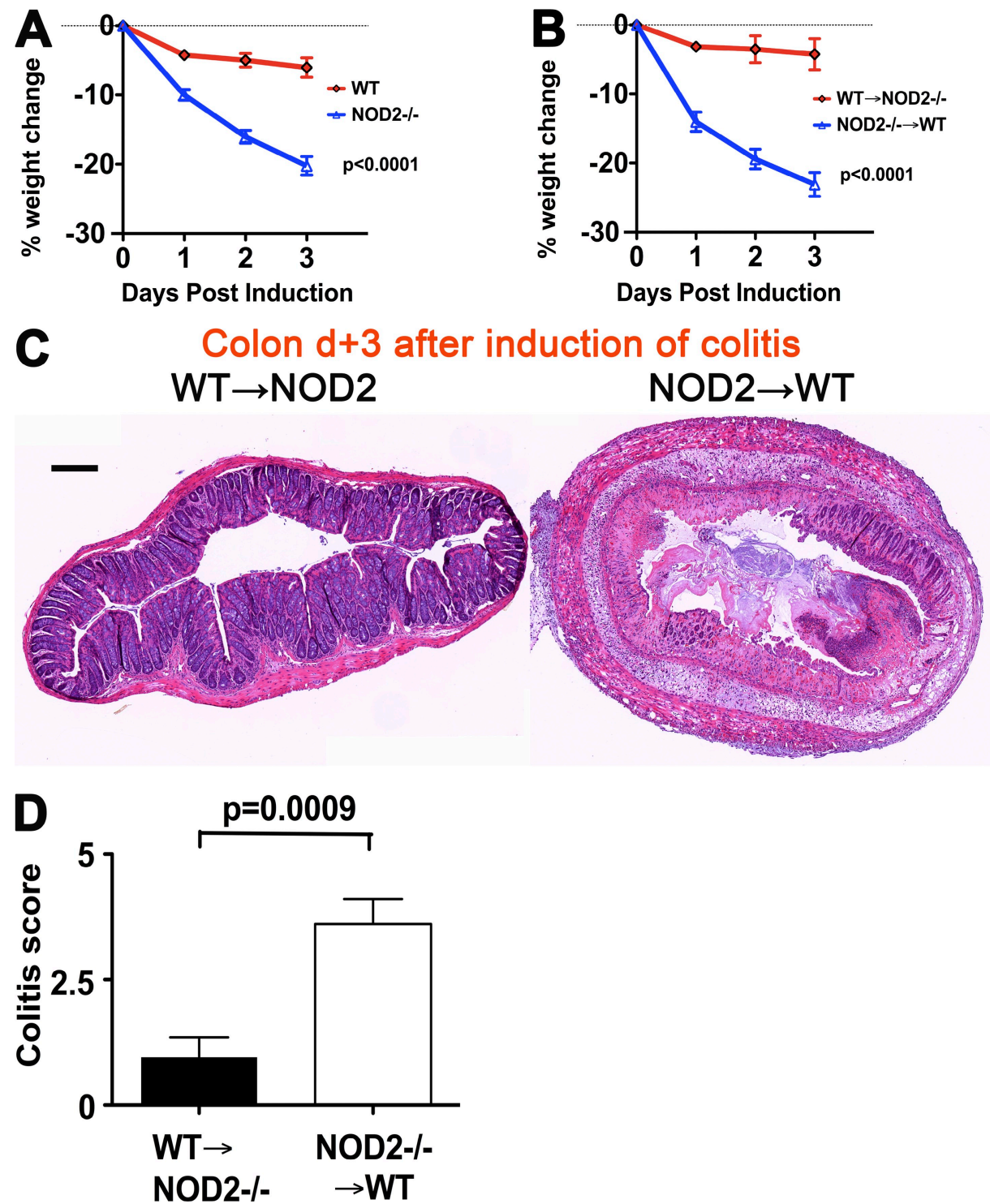

Figure 5. NOD2 deficiency in the hematopoietic system regulates experimental colitis. (A) TNBS colitis (5 mg TNBS in 50\% ethanol) was induced in B6 WT or B6 NOD2 ${ }^{-1-}$ mice. Higher weight loss of NOD2 ${ }^{-1-}$ mice during colitis. Combined data from three independent experiments are shown; $n=12 /$ group. (B-D) First, chimeric mice with NOD2 deficiency either in the hematopoietic system or in the nonhematopoietic system were created by syngeneic BMT (B6 WT $\rightarrow$ B6 NOD2 $2^{-1-}$ or B6 NOD2 ${ }^{-1-} \rightarrow$ B6 WT). After 90 d, TNBS colitis (5 mg TNBS in 50\% ethanol) was induced. Colons were harvested at day 3 after induction of colitis. Combined data from two independent experiments are shown; $n=8 /$ group. (B) Higher weight loss of NOD2 $2^{-1-} \rightarrow$ WT chimera versus WT $\rightarrow$ NOD2 ${ }^{-1-}$ chimera during colitis. (C) Hematoxylin and eosin-stained images of the colon at day 3 after induction of colitis. Bar, $200 \mu$ m. (D) Histological scoring showing increased colitis in the hematopoietic NOD2-deficient chimera. Error bars indicate SEM. 
Induction and assessment of TNBS colitis. Induction of TNBS colitis was performed as published previously (Scheiffele and Fuss, 2002). Severity of colitis was assessed using a semiquantitative scoring system (Scheiffele and Fuss, 2002).

Statistics. Groupwise comparisons were done with the Mann-Whitney test, one-way ANOVA test, or the Wilcoxon matched pairs test where appropriate. Survival data were analyzed with the Mantel-Cox log-rank test.

Online supplemental material. Fig. S1 shows that donor T cell subsets are not different in NOD2 $2^{-/-}$versus WT allo-BMT recipients. In Fig. S2, the donor chimerism at day 90 is given. Fig. $\mathrm{S} 3$ shows serum levels of cytokines and chemokines during GVHD. Fig. S4 demonstrates that NOD2 $2^{-/-}$mice have intact resistance against experimental tumors. Online supplemental material is available at http://www.jem.org/cgi/content/full/jem.20090623/DC1.

We would like to thank Renier Brentjens, Richard Flavell, Ivan J. Fuss, Atsushi Kitani, Katia Manova, Yan Nikhamin, Gabriel Nunez, Eric Pamer, Carles Ubeda-Morant, Joan Zhiqiong Yang, and James Young.

This research was supported by National Institutes of Health grants R01HL069929 (to M. van den Brink), R01-CA107096 (to M. van den Brink), R01Al080455 (to M. van den Brink), R01 HL084815 (to G.F. Murphy), and P01-CA33049 (to M. van den Brink). Support was also received from the Ryan Gibson Foundation, the Elsa U. Pardee Foundation, the Byrne Fund, the Emerald Foundation, the Experimental Therapeutics Center of Memorial Sloan-Kettering Cancer Center funded by Mr. William H. Goodwin and Mrs. Alice Goodwin, the Commonwealth Foundation for Cancer Research, the Bobby Zucker Memorial Fund (to M. van den Brink), the Deutsche Forschungsgemeinschaft (to 0. Penack), the Deutsche Krebshilfe (to I. Na and A. Ghosh), and the Alexander von Humboldt foundation (to K. Brandl).

The authors have no conflicting financial interests.

Submitted: 18 March 2009

Accepted: 11 August 2009

\section{REFERENCES}

Alpdogan, O., S.J. Muriglan, J.M. Eng, L.M. Willis, A.S. Greenberg, B.J. Kappel, and M.R. Van Den Brink. 2003. IL-7 enhances peripheral $\mathrm{T}$ cell reconstitution after allogeneic hematopoietic stem cell transplantation. J. Clin. Invest. 112:1095-1107.

Bertin, J., W.J. Nir, C.M. Fischer, O.V. Tayber, P.R. Errada, J.R. Grant, J.J. Keilty, M.L. Gosselin, K.E. Robison, G.H. Wong, et al. 1999. Human CARD4 protein is a novel CED-4/Apaf-1 cell death family member that activates NF-kappaB. J. Biol. Chem. 274:12955-12958. doi:10.1074/jbc.274.19.12955

Caron, G., D. Duluc, I. Fremaux, P. Jeannin, C. David, H. Gascan, and Y. Delneste. 2005. Direct stimulation of human T cells via TLR5 and TLR7/8: flagellin and R-848 up-regulate proliferation and IFN-gamma production by memory CD4+ T cells. J. Immunol. 175:1551-1557.

Dabbagh, K., and D.B. Lewis. 2003. Toll-like receptors and T-helper-1/ T-helper-2 responses. Curr. Opin. Infect. Dis. 16:199-204.

Elmaagacli, A.H., M. Koldehoff, H. Hindahl, N.K. Steckel, R. Trenschel, R. Peceny, H. Ottinger, P.M. Rath, R.S. Ross, M. Roggendorf, et al. 2006. Mutations in innate immune system NOD2/CARD 15 and TLR-4 (Thr399Ile) genes influence the risk for severe acute graft-versus-host disease in patients who underwent an allogeneic transplantation. Transplantation. 81:247-254. doi:10.1097/01.tp.0000188671.94646.16

Hollander, G.A., B. Widmer, and S.J. Burakoff. 1994. Loss of normal thymic repertoire selection and persistence of autoreactive $\mathrm{T}$ cells in graft vs host disease. J. Immunol. 152:1609-1617.

Holler, E., G. Rogler, H. Herfarth, J. Brenmoehl, P.J. Wild, J. Hahn, G. Eissner, J. Scholmerich, and R. Andreesen. 2004. Both donor and recipient NOD2/CARD15 mutations associate with transplant-related mortality and GvHD following allogeneic stem cell transplantation. Blood. 104:889-894. doi:10.1182/blood-2003-10-3543

Holler, E., G. Rogler, J. Brenmoehl, J. Hahn, H. Herfarth, H. Greinix, A.M. Dickinson, G. Socie, D. Wolff, G. Fischer, et al. 2006. Prognostic significance of NOD2/CARD15 variants in HLA-identical sibling hematopoietic stem cell transplantation: effect on long-term outcome is confirmed in 2 independent cohorts and may be modulated by the type of gastrointestinal decontamination. Blood. 107:4189-4193. doi:10.1182/blood-2005-09-3741

Holler, E., J. Hahn, R. Andreesen, G. Rogler, J. Brenmoehl, H. Greinix, A.M. Dickinson, G. Socie, D. Wolff, and J. Finke. 2008. NOD2/ CARD15 polymorphisms in allogeneic stem-cell transplantation from unrelated donors: T depletion matters. J. Clin. Oncol. 26:338-339. doi:10.1200/JCO.2007.14.1325

Hugot, J.P., M. Chamaillard, H. Zouali, S. Lesage, J.P. Cezard, J. Belaiche, S. Almer, C. Tysk, C.A. O'Morain, M. Gassull, et al. 2001. Association of NOD2 leucine-rich repeat variants with susceptibility to Crohn's disease. Nature. 411:599-603. doi:10.1038/35079107

Inohara, N., and G. Nunez. 2003. NODs: intracellular proteins involved in inflammation and apoptosis. Nat. Rev. Immunol. 3:371-382. doi:10.1038/nri1086

Inohara, N., Y. Ogura, F.F. Chen, A. Muto, and G. Nunez. 2001. Human Nod1 confers responsiveness to bacterial lipopolysaccharides. J. Biol. Chem. 276:2551-2554. doi:10.1074/jbc.M009728200

Kanazawa, N., I. Okafuji, N. Kambe, R. Nishikomori, M. Nakata-Hizume, S. Nagai, A. Fuji, T. Yuasa, A. Manki, Y. Sakurai, et al. 2005. Earlyonset sarcoidosis and CARD15 mutations with constitutive nuclear factor-kappaB activation: common genetic etiology with Blau syndrome. Blood. 105:1195-1197. doi:10.1182/blood-2004-07-2972

Kobayashi, K.S., M. Chamaillard, Y. Ogura, O. Henegariu, N. Inohara, G. Nunez, and R.A. Flavell. 2005. Nod2-dependent regulation of innate and adaptive immunity in the intestinal tract. Science. 307:731-734. doi:10.1126/science.1104911

MacLeod, H., and L.M. Wetzler. 2007. T cell activation by TLRs: a role for TLRs in the adaptive immune response. Sci. STKE. 2007:pe48. doi:10.1126/stke.4022007pe48

Maeda, S., L.C. Hsu, H. Liu, L.A. Bankston, M. Iimura, M.F. Kagnoff, L. Eckmann, and M. Karin. 2005. Nod2 mutation in Crohn's disease potentiates NF-kappaB activity and IL-1beta processing. Science. 307:734-738. doi:10.1126/science. 1103685

Mayor, N.P., B.E. Shaw, D.A. Hughes, H. Maldonado-Torres, J.A. Madrigal, S. Keshav, and S.G. Marsh. 2007. Single nucleotide polymorphisms in the NOD2/CARD15 gene are associated with an increased risk of relapse and death for patients with acute leukemia after hematopoietic stem-cell transplantation with unrelated donors. J. Clin. Oncol. 25:4262-4269. doi:10.1200/JCO.2007.12.1897

Miceli-Richard, C., S. Lesage, M. Rybojad, A.M. Prieur, S. ManouvrierHanu, R. Hafner, M. Chamaillard, H. Zouali, G. Thomas, and J.P. Hugot. 2001. CARD15 mutations in Blau syndrome. Nat. Genet. 29:19-20. doi:10.1038/ng720

Ogura, Y., D.K. Bonen, N. Inohara, D.L. Nicolae, F.F. Chen, R. Ramos, H. Britton, T. Moran, R. Karaliuskas, R.H. Duerr, et al. 2001. A frameshift mutation in NOD2 associated with susceptibility to Crohn's disease. Nature. 411:603-606. doi:10.1038/35079114

Scheiffele, F., and I.J. Fuss. 2002. Induction of TNBS colitis in mice. Curr. Protoc. Immunol. Chapter 15: Unit 15.19.

Strober, W., P.J. Murray, A. Kitani, and T. Watanabe. 2006. Signalling pathways and molecular interactions of NOD1 and NOD2. Nat. Rev. Immunol. 6:9-20. doi:10.1038/nri1747

Terwey, T.H., T.D. Kim, A.A. Kochman, V.M. Hubbard, S. Lu, J.L. Zakrzewski, T. Ramirez-Montagut, J.M. Eng, S.J. Muriglan, G. Heller, et al. 2005. CCR2 is required for CD8-induced graft-versus-host disease. Blood. 106:3322-3330. doi:10.1182/blood-2005-05-1860

van Heel, D.A., S. Ghosh, M. Butler, K.A. Hunt, A.M. Lundberg, T. Ahmad, D.P. Mcgovern, C. Onnie, K. Negoro, S. Goldthorpe, et al. 2005. Muramyl dipeptide and toll-like receptor sensitivity in NOD2-associated Crohn's disease. Lancet. 365:1794-1796. doi:10.1016/S0140-6736(05)66582-8

Watanabe, T., A. Kitani, P.J. Murray, and W. Strober. 2004. NOD2 is a negative regulator of Toll-like receptor 2-mediated T helper type 1 responses. Nat. Immunol. 5:800-808. doi:10.1038/ni1092

Watanabe, T., A. Kitani, P.J. Murray, Y. Wakatsuki, I.J. Fuss, and W. Strober. 2006. Nucleotide binding oligomerization domain 2 deficiency leads to dysregulated TLR 2 signaling and induction of antigen-specific colitis. Immunity. 25:473-485. doi:10.1016/j.immuni.2006.06.018 
Watanabe, T., N. Asano, P.J. Murray, K. Ozato, P. Tailor, I.J. Fuss, A. Kitani, and W. Strober. 2008. Muramyl dipeptide activation of nucleotide-binding oligomerization domain 2 protects mice from experimental colitis. J. Clin. Invest. 118:545-559.

Yang, Z., I.J. Fuss, T. Watanabe, N. Asano, M.P. Davey, J.T. Rosenbaum, W. Strober, and A. Kitani. 2007. NOD2 transgenic mice exhibit enhanced MDP-mediated down-regulation of TLR2 responses and resistance to colitis induction. Gastroenterology. 133:1510-1521. doi:10.1053/j.gastro.2007.07.025
Zakrzewski, J.L., A.A. Kochman, S.X. Lu, T.H. Terwey, T.D. Kim, V.M. Hubbard, S.J. Muriglan, D. Suh, O.M. Smith, J. Grubin, et al. 2006 Adoptive transfer of $\mathrm{T}$-cell precursors enhances $\mathrm{T}$-cell reconstitution after allogeneic hematopoietic stem cell transplantation. Nat. Med. 12:1039-1047. doi:10.1038/nm1463

Zakrzewski, J.L., D. Suh, J.C. Markley, O.M. Smith, C. King, G.L. Goldberg, R. Jenq, A.M. Holland, J. Grubin, J. Cabrera-Perez, et al. 2008. Tumor immunotherapy across MHC barriers using allogeneic T-cell precursors. Nat. Biotechnol. 26:453-461. doi:10.1038/nbt1395 